\title{
Therapeutic Effects of Medicinal Plants on Cutaneous Wound Healing in Humans: A Systematic Review
}

\author{
Tarcisio Vitor Augusto Lordani $\mathbb{D}^{1},{ }^{1}$ Celia Eliane de Lara, ${ }^{1}$ Fabiana Borges Padilha Ferreira, ${ }^{2}$ \\ Mariana de Souza Terron Monich, ${ }^{2}$ Claudinei Mesquita da Silva, ${ }^{2}$ \\ Claudia Regina Felicetti Lordani, ${ }^{3}$ Fernanda Giacomini Bueno, ${ }^{4}$ \\ Jorge Juarez Vieira Teixeira, ${ }^{5}$ and Maria Valdrinez Campana Lonardoni ${ }^{5}$ \\ ${ }^{1}$ Postgraduate Program in Bioscience and Pathophysiology, Universidade Estadual de Maringá, Avenida Colombo 5790, \\ Jardim Universitário, 87020-900 Maringá, PR, Brazil \\ ${ }^{2}$ Postgraduate Program in Health Sciences, Universidade Estadual de Maringá, Avenida Colombo 5790, Jardim Universitário, \\ 87020-900 Maringá, PR, Brazil \\ ${ }^{3}$ Department of Clinical Nutrition, Universidade Estadual do Oeste do Paraná, Rua Universitária 1619, Universitário, \\ 85819-110 Cascavel, PR, Brazil \\ ${ }^{4}$ Medical and Pharmaceutical Science Center, Universidade Estadual do Oeste do Paraná, Rua Universitária 1619, Universitário, \\ 85819-110 Cascavel, PR, Brazil \\ ${ }^{5}$ Department of Clinical Analysis and Biomedicine, Universidade Estadual de Maringá, Avenida Colombo 5790, \\ Jardim Universitário, 87020-900 Maringá, PR, Brazil
}

Correspondence should be addressed to Tarcisio Vitor Augusto Lordani; tarcisiolordani@hotmail.com

Received 29 September 2017; Accepted 2 January 2018; Published 1 April 2018

Academic Editor: Giuseppe Valacchi

Copyright (c) 2018 Tarcisio Vitor Augusto Lordani et al. This is an open access article distributed under the Creative Commons Attribution License, which permits unrestricted use, distribution, and reproduction in any medium, provided the original work is properly cited.

The pharmaceutical industry has made great strides in providing drugs that are able to stimulate the healing process, but only $1-3 \%$ of all drugs that are listed in Western pharmacopoeias are intended for use on the skin or cutaneous wounds. Of these, at least onethird are obtained from plants. We sought to review the therapeutic effects of medicinal plants on human skin lesions. For this systematic review, we searched the PubMed, Scopus, and Web of Science databases to identify clinical trials that were published from 1997 to 2017. We reviewed studies that described the use of medicinal plants for the treatment of skin lesions in humans. Ten studies were selected, eight of which were published from 2007 to 2016, with a total of 503 patients. Among the plant species that were used for the treatment of human skin lesions, 12 belonged to 11 families and were included in the analysis. All of the plant species that were studied presented high therapeutic potential for the treatment of cutaneous lesions.

\section{Introduction}

Wounds are physical injuries that result in the opening or rupture of skin, which can cause anatomical and functional disorders. Skin wounds result in the loss of continuity of the epithelium with or without the loss of underlying connective tissue [1]. Causal factors, signaling inhibitors, preexisting conditions, and the type of injury cumulatively determine whether the healing process will be acute or chronic [2].
Wound healing is a dynamic, complex process that leads to the reestablishment of tissue integrity and homeostasis [3] and involves inflammation, reepithelization, granulated tissue formation, neovascularization, wound contraction, and remodeling of the extracellular matrix [4]. This process is coordinated by a complicated signaling mechanism that involves various growth factors, cytokines, and chemokines. Cell proliferation is a necessary step in tissue repair and regeneration during the wound healing process [5]. These 
injuries constitute a serious public health problem. In the United States, such cutaneous injuries affect thousands of patients and cost billions of dollars to treat [6]. In Brazil, they are also a serious public health problem, although medical records of such injuries are scarce [7].

Wounds that present impaired healing, including acute wounds and chronic wounds, usually do not follow normal physiological healing processes. Such wounds often enter a state of pathological inflammation because of a delayed, incomplete, or uncoordinated healing process [8]. Most chronic wounds are ulcers that are associated with circulatory diseases and diabetes mellitus. Nearly 6 million people suffer from chronic wounds worldwide [9]. In clinical practice, wound dressings and topical products are used to create and maintain a moist environment and provide adequate conditions for healing [10]. However, they are often expensive or ineffective and may generate adverse reactions [11]. Although various pharmaceutical preparations are available, new therapeutic options with fewer adverse effects, a lower cost, and a shorter healing time are continually needed for clinical treatment.

For centuries, plants have been used in both traditional and popular medicine to treat and prevent diseases. In India, "Ayurveda" has been practiced for more than 5000 years as a natural treatment system to prevent and cure diseases, and plants are a part of this process. Traditional Chinese medicine, which is used throughout eastern Asia, is at least 3000 years old and employs numerous plant species [12].

Animal studies of various plant species have generated promising results. For example, Leea macrophylla has healing effects. It increases the synthesis of collagen, stimulates the production of antioxidants, reduces the levels of proinflammatory factors, and improves cell proliferation [13]. Wrightia tinctoria presented healing activity, with an increase in the rate of contraction of induced lesions [14]. Pereskia aculeata accelerated the cicatricial process by increasing blood flow and collagen deposition [10]. An ointment from Struthanthus vulgaris stimulated the closure of lesions, stimulated the formation of granulated tissue, and stimulated the proliferation and organization of collagen fibers [15]. Cynodon dactylon presented antioxidative activity and stimulated collagen formation and healing [16]. Caesalpinia mimosoides stimulated reepithelialization of the epidermal layer and the contraction of lesions [17].

In modern science, plant species that are traditionally used to cure diseases have been extensively studied to identify their bioactive constituents and develop new drugs. Studies of the mechanisms of action and efficacy of these plant compounds have shown that many are pharmacologically safe, warranting further tests in preclinical studies and clinical trials. However, a significant proportion of the world's population cannot afford these modern medicines, and the use of herbal remedies can benefit these patient groups [18].

Considering the importance of the effects of plants and their components on cutaneous tissue, we performed a systematic review to explore the therapeutic effects of medicinal plants on the process of healing cutaneous lesions in humans.

\section{Materials and Methods}

This review was conducted in accordance with the recommendations outlined in the Preferred Reporting Items for Systematic Reviews and Meta-Analyses (PRISMA) statement [19]. The search strategy is described in Figure 1.

2.1. Search Strategy. A systematic review of the literature was performed using the PubMed, Scopus, and Web of Science databases to search for articles that were published from January 1, 1997, to August 31, 2017. This research was structured according to the characteristics of each electronic database. For the retrieval of publications in PubMed and Scopus, Medical Subject Headings (MeSH) terms were used. Four independent researchers (group 1: TVAL, MT, FBF, and CEL) conducted a specific search to define the maximum $\mathrm{MeSH}$ terms that were related to the research goal. Discrepancies were resolved by consensus among the researchers at several meetings. The researchers then presented the $\mathrm{MeSH}$ terms to two specialists (JJVT and MVLC) for validation. In the first phase of the study, four researchers performed the search of the databases and analyzed the titles and abstracts. In PubMed, the MeSH terms were organized into three groups: group 1 (Wound healing OR Re-epithelialization OR Regeneration), group 2 (Medicinal plants OR Phytotherapy OR Plant extract), and group 3 (Cytokines OR Collagen OR Fibroblasts OR Inflammation). The blocks were combined separately to allow the largest number of publications (Block 1 AND Block 2 AND Block 3). To identify publications in the Web of Science database, we searched by topics according to the same three-group structure as in PubMed, which guaranteed the identification of studies with high sensitivity.

\subsection{Study Selection}

2.2.1. Inclusion Criteria. Publications that described the use of medicinal plants for wound healing in humans were included in the systematic review. We considered original studies that were published in English, Spanish, or Portuguese within the predefined period of time and for which abstracts were available in the database.

2.2.2. Exclusion Criteria. Review studies, comparison studies, editor's comments, letters, interviews, guidelines, errata, and articles that did not meet the inclusion criteria were excluded.

2.3. Quality Assessment. For the second phase of the study, based on publications that were previously defined by the researchers in group 1, the full-text articles were retrieved in PDF format. The articles were randomly and proportionally distributed to the researchers. This step was necessary to determine whether the publications would be retained in the systematic review. Disagreements among researchers were resolved by consensus. In the third phase, the selected papers were randomized and distributed to four independent judges (group 2: MVCL, CMS, CRF, and FGB). This phase is considered highly important because it allows the validation of articles that were selected by the researchers in group 1 in accordance with the inclusion criteria. To increase the 


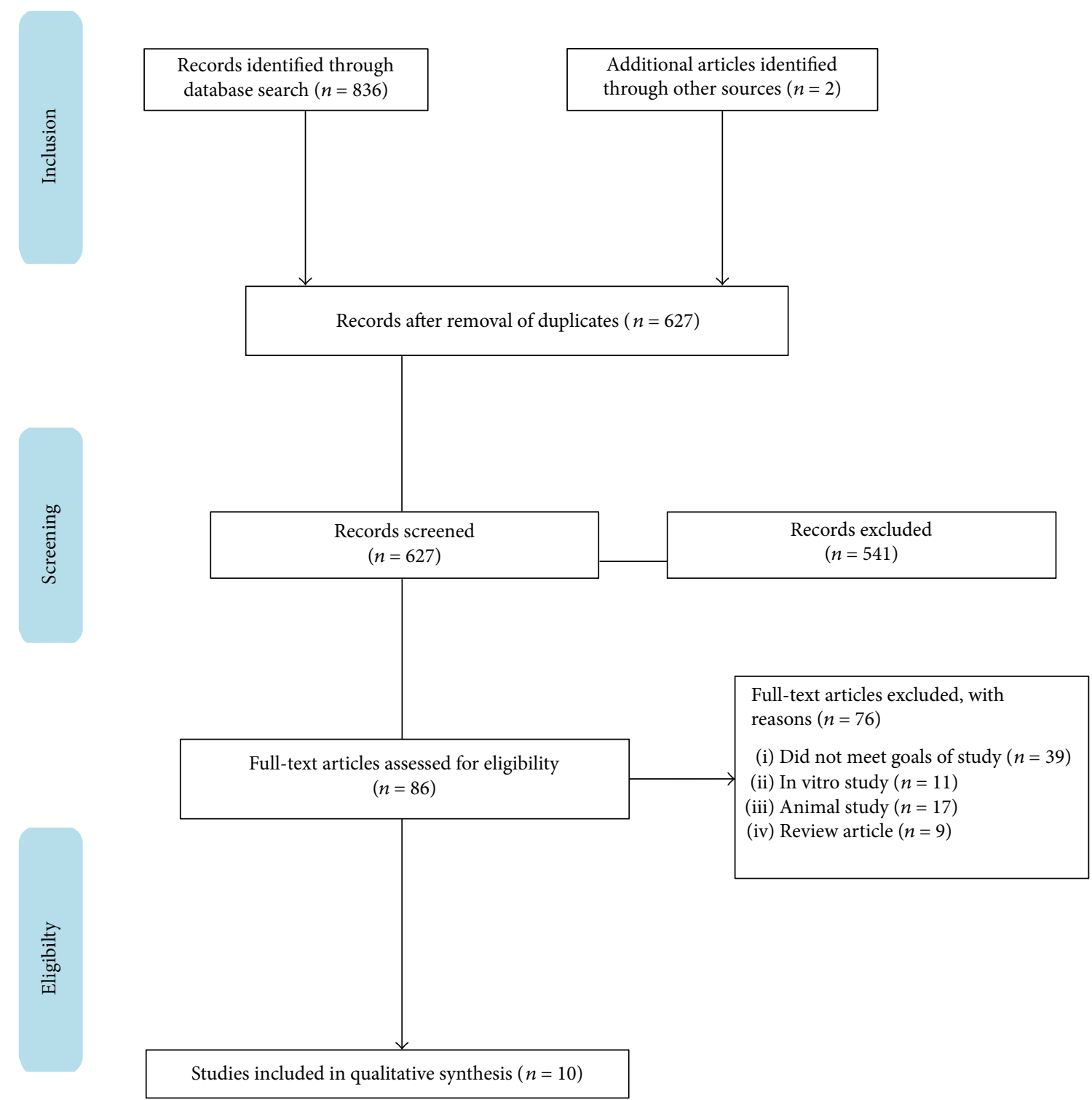

Figure 1: Study flow chart (PRISMA).

sensitivity of this systematic review, we also evaluated the references of the original articles to identify other publications of interest that were not retrieved in the previous database search phases.

2.4. Data Extraction. The fourth phase of the research involved data extraction. The four researchers from group 1 with the support of two specialists (JVT and MVLC) organized the structure of the topics to compile the main findings of the publications. The following data are presented in Table 1: reference, year, country, study type, sample, plant family, scientific name, popular name, route of administration, and treatment outcome. The articles were again randomly assigned to the researchers in group 1 to maintain the completeness of the results. The researchers in group 1 then provided the results to the researchers in group 2 for validation. Any discrepancies were resolved by consensus.

\section{Results}

3.1. Literature Review. A total of 838 abstracts/citations were identified for preliminary review from electronic and manual searches. The primary search identified 836 articles, with 276 from PubMed, 270 from Scopus, 290 from Web of Science, and two from manual searches of the bibliographies. After removal of duplicates and screening for relevant titles and abstracts, a total of 86 articles underwent a full-text review. Ten articles met the inclusion and exclusion criteria. A flow chart that illustrates the study selection and number of articles at each stage is shown in Figure 1.

3.2. Study Characteristics. A total of 503 subjects were evaluated in the 10 studies. The countries where the studies were conducted included the United States $(20 \%, n=2)$, China $(20 \%, n=2)$, Brazil $(20 \%, n=2)$, Mexico $(20 \%, n=2)$, France $(10 \%, n=1)$, and Iran $(10 \%, n=1)$. Eighty percent $(n=8)$ of 


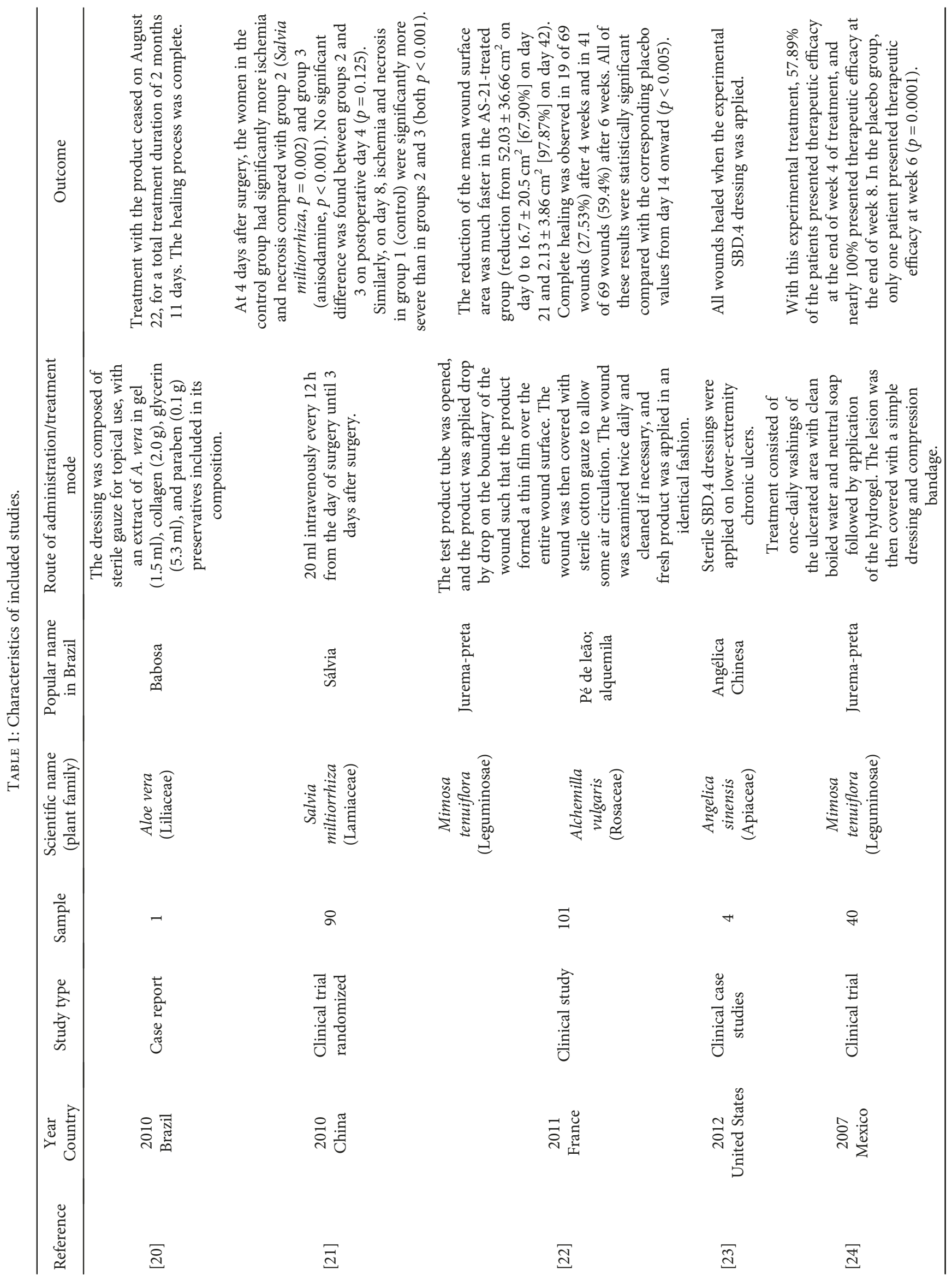




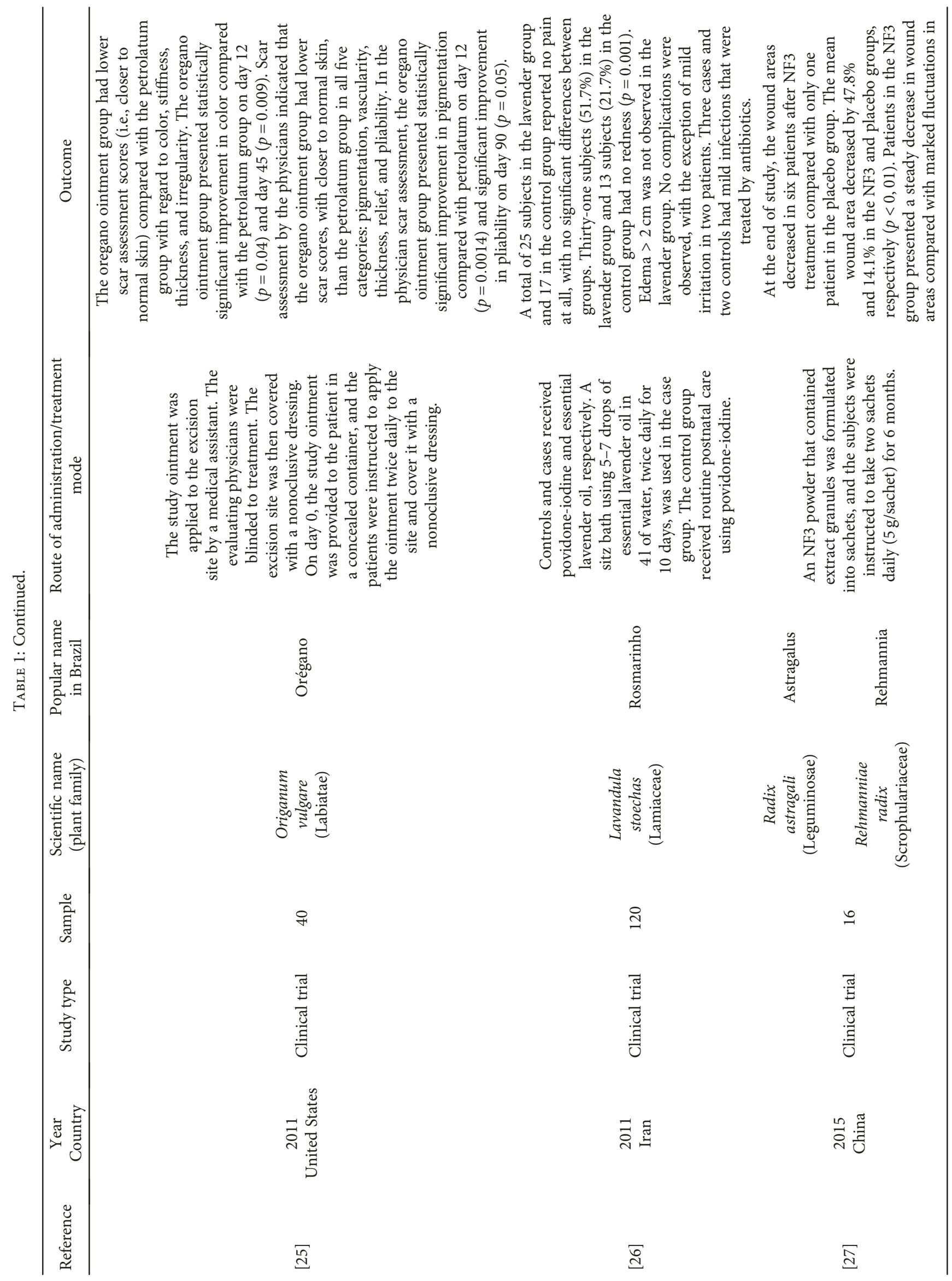




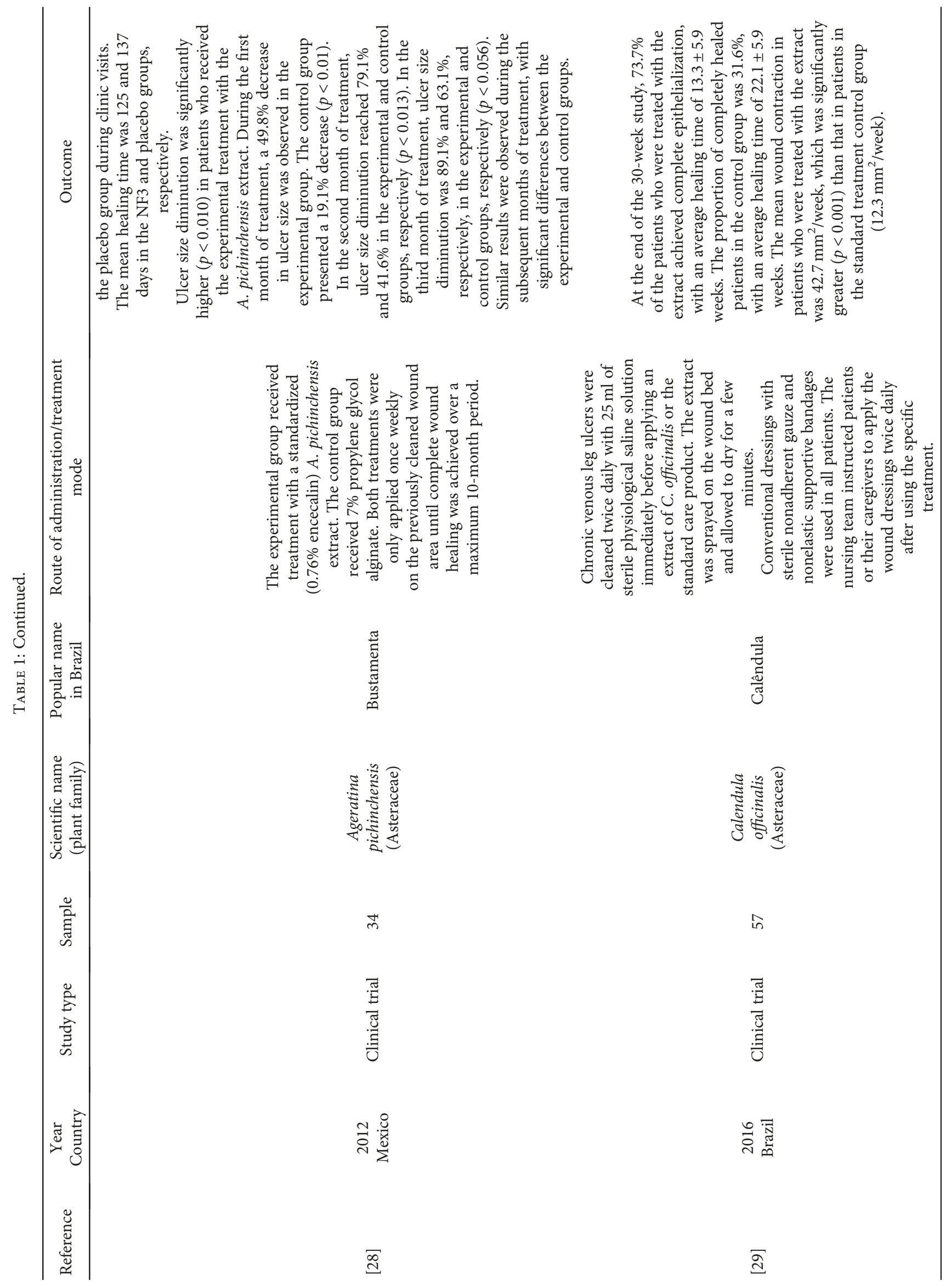


the articles were clinical trials. The studies were published between 2007 and 2016, and most (30\%,n=3) were published in 2011. Regarding the route of administration and treatment mode of the products that were used for wound healing in the selected studies, the topical route of administration was the most commonly used $(80 \%, n=8)$, followed by intravenous $(10 \%, n=1)$ and orogastric $(10 \%$, $n=1$; Table 1).

\section{Discussion}

A notable finding was the scarcity of studies that evaluated the healing effects of medicinal plants in humans in the last decade. The few published studies reported important results for the use of medicinal plants, considering their high therapeutic efficacy [20-29].

Recent years have seen the exponential growth of the use of herbal remedies. These agents are gaining popularity in both developed and developing countries because of their relatively low cost, natural origin, and fewer adverse effects [30]. Numerous drugs that are now used in conventional medicine were originally derived from plants [31]. The present systematic review identified 12 plants with healing actions. Among these plants, Aloe vera, which is native to Brazil, has been used for thousands of years in folk medicine for the treatment of various diseases.

4.1. Aloe vera. The $A$. vera plant has proven pharmacological actions, including healing properties, protection of the skin, and anti-inflammatory and regenerative effects [32]. A. vera has been used for therapeutic purposes by several cultures because of its cicatrizing effects [33]. This plant has a heterogeneous composition. Its gel contains more than 75 bioactive compounds [34], such as aloe and aloe-emodin (which are responsible for its anti-inflammatory action) and glucomannan (which influences fibroblast growth factor, stimulating its proliferation) [35]. Extracts of A. vera stimulate the proliferation of various cell types.

Studies have shown that treatment with $A$. vera gel accelerated the lesion healing process $[36,37]$. Increases in wound contraction and collagen synthesis were reported in a 2006 study [38]. A. vera also promoted fibroblast proliferation and was shown to play an important role in remodeling the extracellular matrix during healing [39].

A. vera and collagen were topically applied for the treatment of an ischemic lesion in a patient with arterial hypertension and diabetes mellitus. At the end of approximately 10 weeks of treatment, the lesion completely healed. The topical application of $A$. vera provides more oxygen, increases vascularity, and increases collagen production, thus promoting wound healing. It also promotes the multiplication of epithelial cells and remodels and heals the lesion [40].

4.2. Salvia miltiorrhiza. S. miltiorrhiza (Salvia) has an Asian origin. It is widely used in traditional Chinese medicine to treat various cardiovascular and cerebrovascular diseases, hyperlipidemia, tumors, and liver problems, such as cirrhosis. It may also influence the recovery of damaged tissue [41]. More than 70 compounds were identified in Salvia, and many of these bioactivities were attributed to two main groups of compounds: salvianolic acids and tanshinones $[42,43]$. The tanshinone group has been extensively studied and reported to have several pharmacological actions, such as anti-inflammatory and antioxidant effects [44].

S. miltiorrhiza improves the healing process by removing necrotic cells, attenuating the inflammatory response, decreasing apoptosis [45, 46], reducing oxidative stress [45], and possibly facilitating the repair of tubular epithelial structures. A rat study evaluated a transgenic S. miltiorrhiza extract that was applied topically to burn wounds, which increased fibroblast growth factor 1 activity 19.2-fold, increased cell proliferation, accelerated the growth of new blood vessels, and reduced healing time [47]. These results corroborate those of Chen et al. [21].

4.3. Mimosa tenuiflora. M. tenuiflora is a leguminous tree that is found in the semiarid region of northern Brazil. It is used medicinally for its curative properties. The stem bark and roots of this tree are widely used by indigenous tribes for psychoactive and medicinal purposes [48]. Mimosa's potential to heal severe skin ulcers was attributed to its high polyphenol content [49] and another group of triterpenic saponins in its bark, mimonosides A-C [50], which induce the proliferation of human cells in culture and have immunomodulatory effects [51]. A Mimosa bark extract was used to treat varicose ulcers and had antimicrobial actions in vitro against a large group of microorganisms [24]. In association with $M$. tenuiflora, the effects of Alchemilla vulgaris were evaluated by Shrivastava [22] in a clinical trial.

4.4. Alchemilla vulgaris. A. vulgaris originated in northern Europe and the mountainous regions of southern Europe. It is commonly known for its astringent and anti-inflammatory properties. It is traditionally used to treat ulcers, eczema, and dermatitis. Similar to other members of the Rosaceae family, A. vulgaris contains polyphenols that are responsible for the major pharmacological actions of the plant. Its extract is rich in proanthocyanidins, which are multiple polymers that are composed of anthocyanidin [52] and have an affinity for proteins $[53,54]$. Procyanidins have been shown to have a strong affinity for proteolytic enzymes, such as elastase, xanthine oxidase, $\beta$-glucuronidase, collagenase, and hyaluronidase, which are involved in the destruction of cell matrix components [52, 55].

Potential wound healing properties have been described for the major components of $A$. vulgaris $[56,57]$. The healing effects of this plant on cutaneous lesions were tested in rats. The results showed healing properties that were associated with the promitotic activity of epithelial cells and myofibroblasts [58].

4.5. Angelica sinensis. A. sinensis originated in Asia and has been used in traditional Chinese medicine for more than 2000 years, mainly to treat gynecological problems, anemia, and wounds $[59,60]$. An Angelica extract induced the cicatrization process, which appeared to occur through multiple actions, such as inhibition of the production of reactive oxygen species (ROS), an increase in cell mobility, the promotion 
of glycolysis, the inhibition of apoptosis, and an increase in the proliferation of fibroblasts [61]. An in vivo study evaluated an SBD.4 isolate from Angelica and found significant increases in healing strength, tissue regeneration, and type I collagen [23]. A. sinensis polysaccharide was shown to have a healing effect on experimental gastric ulcers and an in vitro stimulating effect on the proliferation of gastric epithelial cells [62].

4.6. Origanum vulgare L. O. vulgare L., commonly known as oregano, is native to the entire Mediterranean region, Euro-Siberian region, and Irano-Turanian region. Its essential oils have been used since ancient times to relieve various ailments, such as coughs, colds, skin conditions, and digestive disorders $[63,64]$. The main constituents of the extract of this plant include hydrocarbons, monoterpenes, and phenolic compounds. Carvacrol and thymol are the major products of this plant and are responsible for its antioxidant and antibacterial actions [65]. It also has potential anti-inflammatory effects by inhibiting lipoxygenase and acetylcholinesterase [66].

A rich essential oil of this plant consists of monoterpenes and sesquiterpenes (e.g., caryophyllene and spatulenol), which have antioxidant, antifungal, and antimicrobial effects against various types of bacteria, fungi, and yeast $[67,68]$. Another bioactive constituent of $O$. vulgare is protocatechuic acid, which has high antioxidant capacity and is able to eliminate free radicals by inhibiting lipid peroxidation and suppressing ROS [69]. An in vivo study of titanium dioxide nanoparticles that contained a leaf extract of $O$. vulgare reported significant skin healing activity [70].

4.7. Lavandula stoechas L. L. stoechas L. is a small aromatic shrub that is found in southwestern Europe, the Middle East, and North Africa. It is widely used in traditional medicine to treat various diseases. The essential oil of lavender has beneficial effects on wound healing [71-73]. Clinical trials have suggested a beneficial effect of lavender oil on wound healing. Topical treatment with lavender oil significantly reduced the size of foot-and-mouth ulcerations compared with controls [72]. In another study, wound closure progressed more rapidly with topical application of lavender oil compared with controls, accompanied by an increase in the expression of platelet-derived growth factor-A and epidermal growth factor, thus demonstrating its beneficial effects on tissue remodeling and reepithelialization [73].

L. stoechas L. essential oil has antioxidant, broadspectrum antimicrobial, antifungal, and anti-inflammatory actions [74]. Algieri et al. reported the anti-inflammatory effect of a hydroalcoholic extract of L. stoechas L. in both in vitro and in vivo assays [75]. The effects of a 10\% extract of $L$. stoechas L. on open lesions were evaluated in rats, with greater lesion contraction and significant histological results compared with controls [76].

L. stoechas L. oil had positive effects on wound healing in rats and was recommended for the treatment of chronically infected wounds because of its immunostimulating action and antimicrobial effects [77]. However, to our knowledge, only one study has evaluated its healing effects in humans [26].

4.8. Radix astragali. R. astragali is a well-known herb in traditional Chinese medicine. The main constituents of $R$. astragali include polysaccharides, saponins, flavonoids, amino acids, and trace elements [78]. A saponin- and isoflavoneenriched extract of $R$. astragali promoted angiogenesis in human endothelial cells [79], and calicossin is known as an angiogenesis-promoting isoflavone [80]. Recent studies have shown that flavonoids in $R$. astragali have strong antioxidant activity and pharmacological effects, such as mitigation of the deleterious effects of hypoxia [81] and anti-inflammatory, antioxidative, immunoregulatory, and neuroprotective actions [82].

A randomized, double-blind, placebo-controlled study of two herbal formulations found that they effectively promoted the healing of chronic diabetic ulcers, with recovery in $85 \%$ of patients [83]. Among the herbal components, $R$. astragali and Rehmanniae radix were shown to have the most pronounced effects. These two species were shown to stimulate the growth of fibroblasts in diabetic foot ulcer tissue in diabetic patients $[84,85]$.

4.9. Rehmanniae radix. $R$. radix is classified as a very effective herb in traditional Chinese medicine. It consists of approximately 70 monomers. Polysaccharides, oligosaccharides, stachyose, and monosaccharides have been identified in $R$. radix in high concentrations [86]. Recent studies have shown that $R$. radix and its active constituents have a wide spectrum of pharmacological actions in the circulatory, immune, endocrine, cardiovascular, and nervous systems [87]. Its aqueous extract stimulated the proliferation of fibroblasts and effectively healed diabetic foot ulcers in rats through tissue regeneration, angiogenesis, and inflammation control processes $[84,85]$.

4.10. Ageratina pichinchensis. A. pichinchensis is a member of the Asteraceae family. It has been used for many years for the treatment of cutaneous lesions. Preliminary pharmacological tests of extracts of $A$. pichinchensis revealed a shorter time of cicatrization of surgically induced cutaneous wounds in rats [88]. One study identified and isolated the flavonoid 7-O( $\beta$-D-glucopyranosyl)-galactin, to which the cell proliferation capacity of human skin was attributed [89].

A double-blind, controlled clinical study evaluated the effects of topical application of a pharmaceutical formulation that contained an extract of $A$. pichinchensis in patients with chronic venous leg ulcers, and 100\% therapeutic efficacy was found [28]. Antimicrobial and anti-inflammatory effects [89] of extracts of this species have also been described, which may contribute to its wound healing effects.

4.11. Calendula officinalis. The most recent study that was identified in the present systematic review evaluated $C$. officinalis L. This plant has been cultivated in Europe for centuries and is used medicinally throughout the world [90]. The main constituents of $C$. officinalis are pentacyclic triterpenes, including $\psi$-taraxastene, taraxastene, lupene, $\Delta 12$-oleanene, and $\Delta 12$-ursene [91]. These triterpenes exist as monols, diols, 
and triols in free form, esterified with long fatty acids or as oleanene-type saponins. Triterpenes in C. officinalis flowers can balance the inflammatory response and may be beneficial for the treatment of chronic wounds that are associated with chronic inflammation [92]. A 5\% marigold tincture was shown to have cicatrization actions [93], with an increase in cells that are involved in the cicatricial process, resulting in a more satisfactory healing response in rabbits, compared with other treatments that were applied to experimental skin wounds. C. officinalis treatment in patients with venous ulcers accelerated healing [94]. Leach [95] performed a systematic review of several clinical trials and concluded that C. officinalis has several properties that are conducive to wound healing.

Medicinal plants are important alternatives for the healing of lesions. Their use has been validated in clinical trials that evaluated their beneficial and adverse effects. Further studies are needed to demonstrate their clinical efficacy and safety and evaluate costs and benefits.

One limitation of the present study was that we searched only three databases. Another limitation was that we only evaluated studies that were published in English, Spanish, and Portuguese. Furthermore, we only included papers that had summaries/abstracts available online.

\section{Conclusions}

A total of 12 medicinal plants and herbal compounds have been studied in clinical trials and case studies with regard to their wound healing actions. Only one plant species was used in more than one publication (by different authors). These 12 plants were reported to have high skin lesion healing potential, with significant differences compared with those of controls. Two case studies described the complete healing of lesions in patients. Mimosa tenuiflora was the most studied plant in the last decade. Two studies reported its potential wound healing properties, with $100 \%$ efficacy for the treatment of lesions.

\section{Conflicts of Interest}

The authors declare no conflict of interest.

\section{Authors' Contributions}

Tarcisio Vitor Augusto Lordani, Mariana de Souza Terron Monich, Celia Eliane de Lara, and Fabiana Borges Padilha Ferreira searched the databases and analyzed the data; Maria Valdrinez Campana Lonardoni, Jorge Juarez Vieira Teixeira, and Claudinei Mesquita da Silva reviewed the search results and resolved the discrepancies. Tarcisio Vitor Augusto Lordani, Claudia Regina Felicetti Lordani, and Fernanda Giacomini Bueno wrote the paper.

\section{Acknowledgments}

This research was supported by the Conselho Nacional de Desenvolvimento Científico e Tecnológico (CNPq), Coordenação de Aperfeiçoamento de Pessoal de Nível Superior
(CAPES), State University of Maringá, and State University of West Paraná.

\section{Supplementary Materials}

Preferred Reporting Items for Systematic Reviews and MetaAnalyses (PRISMA) statement [19]. (Supplementary Materials)

\section{References}

[1] F. Strodtbeck, "Physiology of wound healing," Newborn and Infant Nursing Reviews, vol. 1, no. 1, pp. 43-52, 2001.

[2] S. Schreml, R.-M. Szeimies, L. Prantl, M. Landthaler, and P. Babilas, "Wound healing in the 21st century," Journal of the American Academy of Dermatology, vol. 63, no. 5, pp. 866-881, 2010.

[3] S. A. Eming, T. Krieg, and J. M. Davidson, "Inflammation in wound repair: molecular and cellular mechanisms," Journal of Investigative Dermatology, vol. 127, no. 3, pp. 514-525, 2007.

[4] A. J. Singer and R. A. F. Clark, "Cutaneous wound healing," New England Journal of Medicine, vol. 341, no. 10, pp. 738746, 1999.

[5] W. Xing, W. Guo, C. H. Zou et al., "Acemannan accelerates cell proliferation and skin wound healing through AKT/mTOR signaling pathway," Journal of Dermatological Science, vol. 79, no. 2, pp. 101-109, 2015.

[6] T. A. Mustoe, K. O’Shaughnessy, and O. Kloeters, "Chronic wound pathogenesis and current treatment strategies: a unifying hypothesis," Plastic and Reconstructive Surgery, vol. 117, Supplement, pp. 35S-41S, 2006.

[7] I. C. Santos, M. A. Souza, L. N. Andrade, M. P. Lopes, M. F. Barros e Silva, and R. T. Santiago, "Characterization of care for patients with wounds in primary care," Revista da Rede de Enfermagem do Nordeste, vol. 15, no. 4, pp. 613-620, 2014.

[8] C. Agyare, Y. D. Boakye, E. O. Bekoe, A. Hensel, S. O. Dapaah, and T. Appiah, "Review: African medicinal plants with wound healing properties," Journal of Ethnopharmacology, vol. 177, pp. 85-100, 2016.

[9] N. B. Menke, K. R. Ward, T. M. Witten, D. G. Bonchev, and R. F. Diegelmann, "Impaired wound healing," Clinics in Dermatology, vol. 25, no. 1, pp. 19-25, 2007.

[10] N. de Castro Campos Pinto, P. Cassini-Vieira, E. M. de SouzaFagundes, L. S. Barcelos, M. C. M. N. Castañon, and E. Scio, "Pereskia aculeata Miller leaves accelerate excisional wound healing in mice," Journal of Ethnopharmacology, vol. 194, pp. 131-136, 2016.

[11] A. P. do Nascimento Duque, N. de Castro Campos Pinto, R. de Freitas Mendes et al., "In vivo wound healing activity of gels containing Cecropia pachystachya leaves," The Journal of Pharmacy and Pharmacology, vol. 68, no. 1, pp. 128-138, 2016.

[12] P. Garodia, H. Ichikawa, N. Malani, G. Sethi, and B. B. Aggarwal, "From ancient medicine to modern medicine: Ayurvedic concepts of health and their role in inflammation and cancer," Journal of the Society for Integrative Oncology, vol. 05, no. 1, p. 25, 2007.

[13] A. Joshi, V. K. Joshi, D. Pandey, and S. Hemalatha, "Systematic investigation of ethanolic extract from Leea macrophylla: implications in wound healing," Journal of Ethnopharmacology, vol. 191, pp. 95-106, 2016. 
[14] M. Yariswamy, H. V. Shivaprasad, V. Joshi, A. N. Nanjaraj Urs, A. Nataraju, and B. S. Vishwanath, "Topical application of serine proteases from Wrightia tinctoria R. Br. (Apocyanaceae) latex augments healing of experimentally induced excision wound in mice," Journal of Ethnopharmacology, vol. 149, no. 1, pp. 377-383, 2013.

[15] L. S. dos Santos Gramma, F. M. Marques, C. Vittorazzi et al., "Struthanthus vulgaris ointment prevents an over expression of inflammatory response and accelerates the cutaneous wound healing," Journal of Ethnopharmacology, vol. 190, pp. 319-327, 2016.

[16] T. K. Biswas, S. Pandit, S. Chakrabarti, S. Banerjee, N. Poyra, and T. Seal, "Evaluation of Cynodon dactylon for wound healing activity," Journal of Ethnopharmacology, vol. 197, pp. 128137, 2017.

[17] P. B. Bhat, S. Hegde, V. Upadhya, G. R. Hegde, P. V. Habbu, and G. S. Mulgund, "Evaluation of wound healing property of Caesalpinia mimosoides Lam," Journal of Ethnopharmacology, vol. 193, pp. 712-724, 2016.

[18] S. Prasad and A. K. Tyagi, "Traditional medicine: the goldmine for modern drugs," Advanced Techniques in Biology \& Medicine, vol. 03, no. 1, 2015.

[19] L. Shamseer, D. Moher, M. Clarke et al., "Preferred Reporting Items for Systematic Review and Meta-Analysis protocols (PRISMA-P) 2015: elaboration and explanation," BMJ, vol. 349, 2015.

[20] S. H. dos Santos Oliveira, M. J. G. O. Soares, and P. de Sousa Rocha, "Uso de cobertura com colágeno e Aloe vera no tratamento de ferida isquêmica: estudo de caso," Revista da Escola de Enfermagem da U.S.P., vol. 44, no. 2, pp. 346-351, 2010.

[21] J. Chen, Q. Lv, M. Yu, X. Zhang, and J. Gou, "Randomized clinical trial of Chinese herbal medications to reduce wound complications after mastectomy for breast carcinoma," The British Journal of Surgery, vol. 97, no. 12, pp. 1798-1804, 2010.

[22] R. Shrivastava, "Clinical evidence to demonstrate that simultaneous growth of epithelial and fibroblast cells is essential for deep wound healing," Diabetes Research and Clinical Practice, vol. 92, no. 1, pp. 92-99, 2011.

[23] H. Zhao, J. Deneau, G. O. L. Che et al., "Angelica sinensis isolate SBD.4: composition, gene expression profiling, mechanism of action and effect on wounds, in rats and humans," Dermatology, vol. 22, no. 1, pp. 58-67, 2012.

[24] E. Rivera-Arce, M. A. Chávez-Soto, A. Herrera-Arellano et al., "Therapeutic effectiveness of a Mimosa tenuiflora cortex extract in venous leg ulceration treatment," Journal of Ethnopharmacology, vol. 109, no. 3, pp. 523-528, 2007.

[25] J. Ragi, A. Pappert, B. Rao, D. Havkin-Frenkel, and S. Milgraum, "Oregano extract ointment for wound healing: a randomized, double-blind, petrolatum-controlled study evaluating efficacy," Journal of Drugs in Dermatology, vol. 10, no. 10, pp. 1168-1172, 2011.

[26] K. Vakilian, M. Atarha, R. Bekhradi, and R. Chaman, "Healing advantages of lavender essential oil during episiotomy recovery: a clinical trial," Complementary Therapies in Clinical Practice, vol. 17, no. 1, pp. 50-53, 2011.

[27] C. H. Ko, S. Yi, R. Ozaki et al., "Healing effect of a two-herb recipe (NF3) on foot ulcers in Chinese patients with diabetes: a randomized double-blind placebo-controlled study," Journal of Diabetes, vol. 6, no. 4, pp. 323-334, 2014.

[28] O. Romero-cerecero, A. Zamilpa-álvarez, E. Jiménez-ferrer, and J. Tortoriello, "Exploratory study on the effectiveness of a standardized extract from Ageratina pichinchensis in patients with chronic venous leg ulcers," Planta Medica, vol. 78, no. 04, pp. 304-310, 2012.

[29] M. Buzzi, F. de Freitas, and M. de Barros Winter, "Therapeutic effectiveness of a Calendula officinalis extract in venous leg ulcer healing," Journal of Wound Care, vol. 25, no. 12, pp. 732-739, 2016.

[30] M. Modak, P. Dixit, J. Londhe, S. Ghaskadbi, and T. P. A. Devasagayam, "Indian herbs and herbal drugs used for the treatment of diabetes," Journal of Clinical Biochemistry and Nutrition, vol. 40, no. 3, pp. 163-173, 2007.

[31] I. A. Oreagba, K. A. Oshikoya, and M. Amachree, "Herbal medicine use among urban residents in Lagos, Nigeria," BMC Complementary and Alternative Medicine, vol. 11, no. 1, p. 117, 2011.

[32] I. R. Domínguez, O. S. Gutiérrez, O. R. López, and M. F. Naranjo, "Beneficios del Aloe vera l. (sábila) en las afecciones de la piel," Revista Cubana de Enfermería, vol. 22, no. 3, 2006.

[33] O. M. Grace, M. S. J. Simmonds, G. F. Smith, and A. E. van Wyk, "Therapeutic uses of Aloe L. (Asphodelaceae) in southern Africa," Journal of Ethnopharmacology, vol. 119, no. 3, pp. 604-614, 2008.

[34] L. Langmead, R. J. Makins, and D. S. Rampton, “Anti-inflammatory effects of Aloe vera gel in human colorectal mucosa in vitro," Alimentary Pharmacology \& Therapeutics, vol. 19, no. 5, pp. 521-527, 2004.

[35] M.-Y. Park, H.-J. Kwon, and M.-K. Sung, "Evaluation of aloin and aloe-emodin as anti-inflammatory agents in aloe by using murine macrophages," Bioscience, Biotechnology, and Biochemistry, vol. 73, no. 4, pp. 828-832, 2009.

[36] M. Tarameshloo, M. Norouzian, S. Zarein-Dolab, M. Dadpay, J. Mohsenifar, and R. Gazor, "Aloe vera gel and thyroid hormone cream may improve wound healing in Wistar rats," Anatomy \& Cell Biology, vol. 45, no. 3, pp. 170-177, 2012.

[37] C. Liu, M. Y. K. Leung, J. C. M. Koon et al., "Macrophage activation by polysaccharide biological response modifier isolated from Aloe vera L. var. chinensis (Haw.) Berg," International Immunopharmacology, vol. 6, no. 11, pp. 16341641, 2006.

[38] S. Subramanian, D. Sathish Kumar, and P. Arulselvan, "Wound healing potential of Aloe vera leaf gel studied in experimental rabbits," Asian Journal of Biochemistry, vol. 1, no. 2, pp. 178-185, 2006.

[39] P. Chantarawaratit, P. Sangvanich, and P. Thunyakitpisal, "Acemannan stimulates bone sialoprotein, osteocalcin, osteopontin and osteonectin expression in periodontal ligament cells in vitro," Journal of the Dental Association of Thailand, vol. 63, no. 2, pp. 92-102, 2013.

[40] A. de Paula Ramos and L. C. Pimentel, "Ação da Babosa no reparo tecidual e cicatrização," Brazilian Journal of Health, vol. 2, no. 1, pp. 40-48, 2011.

[41] I. S. Lay, C. C. Hsieh, J. H. Chiu, M. S. Shiao, W. Y. Lui, and $\mathrm{C} . \mathrm{W}$. $\mathrm{Wu}$, "Salvianolic acid $\mathrm{B}$ enhances in vitro angiogenesis and improves skin flap survival in Sprague-Dawley rats," The Journal of Surgical Research, vol. 115, no. 2, pp. 279-285, 2003.

[42] T. O. Cheng, "Cardiovascular effects of Danshen," International Journal of Cardiology, vol. 121, no. 1, pp. 9-22, 2007.

[43] B.-Q. Wang, "Salvia miltiorrhiza: chemical and pharmacological review of a medicinal plant," Journal of Medicinal Plants Research, vol. 4, no. 25, pp. 2813-2820, 2010. 
[44] S. Y. Kim, T. C. Moon, H. W. Chang, K. H. Son, S. S. Kang, and H. P. Kim, "Effects of tanshinone I isolated from Salvia miltiorrhiza Bunge on arachidonic acid metabolism and in vivo inflammatory responses," Phytotherapy Research, vol. 16, no. 7, pp. 616-620, 2002.

[45] H. Q. Yin, Y. J. Choi, Y. C. Kim, D. H. Sohn, S. Y. Ryu, and B. H. Lee, "Salvia miltiorrhiza Bunge and its active component cryptotanshinone protects primary cultured rat hepatocytes from acute ethanol-induced cytotoxicity and fatty infiltration," Food and Chemical Toxicology, vol. 47, no. 1, pp. 98-103, 2009.

[46] X. Yin, Y. Yin, F.-L. Cao et al., "Tanshinone IIA attenuates the inflammatory response and apoptosis after traumatic injury of the spinal cord in adult rats," PLoS One, vol. 7, no. 6, article e38381, 2012.

[47] Y. Tan, K. Y. Wang, N. Wang, G. Li, and D. Liu, "Ectopic expression of human acidic fibroblast growth factor 1 in the medicinal plant, Salvia miltiorrhiza, accelerates the healing of burn wounds," BMC Biotechnology, vol. 14, no. 1, p. 74, 2014.

[48] S. L. Camargo-Ricalde, "Description, distribution, anatomy, chemical composition and uses of Mimosa tenuiflora (Fabaceae-Mimosoideae) in Mexico," Revista de Biología Tropical, vol. 48, no. 4, pp. 939-954, 2000.

[49] E. Rivera-Arce, M. Gattuso, R. Alvarado et al., "Pharmacognostical studies of the plant drug Mimosae tenuiflorae cortex," Journal of Ethnopharmacology, vol. 113, no. 3, pp. 400-408, 2007.

[50] Y. Jiang, G. Massiot, C. Lavaud et al., "Triterpenoid glycosides from the bark of Mimosa tenuiflora," Phytochemistry, vol. 30, no. 7, pp. 2357-2360, 1991.

[51] R. Anton, Y. Jiang, B. Weniger, J. P. Beck, and L. Rivier, "Pharmacognosy of Mimosa tenuiflora (Willd.) Poiret," Journal of Ethnopharmacology, vol. 38, no. 2-3, pp. 145-152, 1993.

[52] R. Facino, M. Carini, G. Aldini, E. Bombardelli, P. Morazzoni, and R. Morelli, "Free radicals scavenging action and antienzyme activities of procyanidines from Vitis vinifera. A mechanism for their capillary protective action," Arzneimittel-Forschung, vol. 44, no. 5, pp. 592-601, 1994.

[53] J. M. Tixier, G. Godeau, A. M. Robert, and W. Hornebeck, "Evidence by in vivo and in vitro studies that binding of pycnogenols to elastin affects its rate of degradation by elastases," Biochemical Pharmacology, vol. 33, no. 24, pp. 3933-3939, 1984.

[54] Y.-J. Zhang, T. Tanaka, Y. Betsumiya et al., "Association of tannins and related polyphenols with the cyclic peptide gramicidin S," Chemical \& Pharmaceutical Bulletin, vol. 50, no. 2, pp. 258-262, 2002.

[55] M. A. Bos, B. Vennat, M. T. Meunier, M. P. Pouget, A. Pourrat, and J. Fialip, "Procyanidins from tormentil: antioxidant properties towards lipoperoxidation and anti-elastase activity," Biological \& Pharmaceutical Bulletin, vol. 19, no. 1, pp. 146-148, 1996.

[56] F. Ashoori, S. Suzuki, J. H. Zhou, N. Isshiki, and Y. Miyachi, "Involvement of lipid peroxidation in necrosis of skin flaps and its suppression by ellagic acid," Plastic and Reconstructive Surgery, vol. 94, no. 7, pp. 1027-1037, 1994.

[57] K. Gomathi, D. Gopinath, M. Rafiuddin Ahmed, and R. Jayakumar, "Quercetin incorporated collagen matrices for dermal wound healing processes in rat," Biomaterials, vol. 24, no. 16, pp. 2767-2772, 2003.
[58] R. Shrivastava, N. Cucuat, and G. W. John, "Effects of Alchemilla vulgaris and glycerine on epithelial and myofibroblast cell growth and cutaneous lesion healing in rats," Phytotherapy Research, vol. 21, no. 4, pp. 369-373, 2007.

[59] P. P. Wang, Y. Zhang, L. Q. Dai, and K. P. Wang, "Effect of Angelica sinensis polysaccharide-iron complex on iron deficiency anemia in rats," Chinese Journal of Integrative Medicine, vol. 13, no. 4, pp. 297-300, 2007.

[60] K.-F. Huang, Y.-C. Hsu, C.-N. Lin, J.-I. Tzeng, Y.-W. Chen, and J.-J. Wang, "Shiunko promotes epithelization of wounded skin," The American Journal of Chinese Medicine, vol. 32, no. 3, pp. 389-396, 2004.

[61] C. Y. Hsiao, C. Y. Hung, T. H. Tsai, and K. F. Chak, "A study of the wound healing mechanism of a traditional chinese medicine, Angelica sinensis, using a proteomic approach," Evidence-Based Complementary and Alternative Medicine, vol. 2012, 14 pages, 2012.

[62] Y. N. Ye, H. L. So, E. S. L. Liu, V. Y. Shin, and C. H. Cho, "Effect of polysaccharides from Angelica sinensis on gastric ulcer healing," Life Sciences, vol. 72, no. 8, pp. 925-932, 2003.

[63] S. J. Ibadullayeva, M. Shahmuradova, M. Gahramanova, and S. G. Aliyeva, "Use of wild plants at dermatosis (skin deseases): ethnobotany," Journal of Applied Pharmaceutical Science, vol. 2, no. 8, pp. 64-67, 2012.

[64] R. Polat and F. Sat1l, "An ethnobotanical survey of medicinal plants in Edremit gulf (Balıkesir-Turkey)," Journal of Ethnopharmacology, vol. 139, no. 2, pp. 626-641, 2012.

[65] J. Coccimiglio, M. Alipour, Z.-H. Jiang, C. Gottardo, and Z. Suntres, "Antioxidant, antibacterial, and cytotoxic activities of the ethanolic Origanum vulgare extract and its major constituents," Oxidative Medicine and Cellular Longevity, vol. 2016, 8 pages, 2016.

[66] A. Carrasco, E. Pérez, A.-B. Cutillas, R. Martinez-Gutierrez, V. Tomas, and J. Tudela, "Origanum vulgare and Thymbra capitata essential oils from Spain: determination of aromatic profile and bioactivities," Natural Product Communications, vol. 11, no. 1, pp. 113-120, 2016.

[67] B. Lukas, C. Schmiderer, and J. Novak, "Essential oil diversity of European Origanum vulgare L. (Lamiaceae)," Phytochemistry, vol. 119, pp. 32-40, 2015.

[68] F. Sahin, M. Güllüce, D. Daferera et al., "Biological activities of the essential oils and methanol extract of Origanum vulgare ssp. vulgare in the Eastern Anatolia region of Turkey," Food Control, vol. 15, no. 7, pp. 549-557, 2004.

[69] T. H. Chou, H. Y. Ding, R. J. Lin, J. Y. Liang, and C. H. Liang, "Inhibition of melanogenesis and oxidation by protocatechuic acid from Origanum vulgare (oregano)," Journal of Natural Products, vol. 73, no. 11, pp. 1767-1774, 2010.

[70] R. Sankar, R. Dhivya, K. S. Shivashangari, and V. Ravikumar, "Wound healing activity of Origanum vulgare engineered titanium dioxide nanoparticles in Wistar albino rats," Journal of Materials Science. Materials in Medicine, vol. 25, no. 7, pp. 1701-1708, 2014.

[71] K. K. Reddy, L. Grossman, and G. S. Rogers, "Common complementary and alternative therapies with potential use in dermatologic surgery: risks and benefits," Journal of the American Academy of Dermatology, vol. 68, no. 4, pp. e127-e135, 2013.

[72] D. T. Altaei, "Topical lavender oil for the treatment of recurrent aphthous ulceration," American Journal of Dentistry, vol. 25, no. 1, pp. 39-43, 2012. 
[73] A. K. Kutlu, D. Çeçen, S. Gül, G. Gürgen, J. OS, and F. Çetin, "A comparison study of growth factor expression following treatment with transcutaneous electrical nerve stimulation, saline solution, povidone-iodine, and lavender oil in wounds healing," Evidence-Based Complementary and Alternative Medicine, vol. 2013, 9 pages, 2013.

[74] S. Arantes, M. F. Candeias, A. Piçarra et al., "Biomarkers and toxicological evaluation of Lavandula spp. essential oils," Exp Pathol Heal Sci Res Clin Teach Soc J Port Exp Pathol Assoc., vol. 7, no. 1, pp. 46-47, 2015.

[75] F. Algieri, A. Rodriguez-Nogales, T. Vezza et al., "Anti-inflammatory activity of hydroalcoholic extracts of Lavandula dentata L. and Lavandula stoechas L," Journal of Ethnopharmacology, vol. 190, pp. 142-158, 2016.

[76] T. A. Çelik and Ö. S. Aslantürk, "Cytotoxic and genotoxic effects of Lavandula stoechas aqueous extracts," Biologia, vol. 62 , no. 3, 2007.

[77] P. E. Lusby, A. L. Coombes, and J. M. Wilkinson, "A comparison of wound healing following treatment with Lavandula $\mathrm{x}$ allardii honey or essential oil," Phytotherapy Research, vol. 20, no. 9, pp. 755-757, 2006.

[78] Q. T. Yu, L. W. Qi, P. Li, L. Yi, J. Zhao, and Z. Bi, "Determination of seventeen main flavonoids and saponins in the medicinal plant Huang-qi (Radix astragali) by HPLC-DAD-ELSD," Journal of Separation Science, vol. 30, no. 9, pp. 1292-1299, 2007.

[79] R. P. Zhang, X. P. Zhang, Y. F. Ruan et al., "Protective effect of Radix astragali injection on immune organs of rats with obstructive jaundice and its mechanism," World Journal of Gastroenterology, vol. 15, no. 23, pp. 2862-2869, 2009.

[80] J. Y. Tang, S. Li, Z. H. Li et al., "Calycosin promotes angiogenesis involving estrogen receptor and mitogen-activated protein kinase (MAPK) signaling pathway in zebrafish and HUVEC," PLoS One, vol. 5, no. 7, p. e11822, 2010.

[81] Y. Fan, D. Z. Wu, Y. Q. Gong, J. Y. Zhou, and Z. B. Hu, "Effects of calycosin on the impairment of barrier function induced by hypoxia in human umbilical vein endothelial cells," European Journal of Pharmacology, vol. 481, no. 1, pp. 33-40, 2003.

[82] J. L. Rios and P. G. Waterman, "A review of the pharmacology and toxicology of Astragalus," Phytotherapy Research, vol. 11, no. 6, pp. 411-418, 1997.

[83] P. C. Leung, M. W. N. Wong, and W. C. Wong, "Limb salvage in extensive diabetic foot ulceration: an extended study using a herbal supplement," Hong Kong Medical Journal, vol. 14, no. 1, pp. 29-33, 2008.

[84] T. W. Lau, Y. W. Chan, C. P. Lau et al., "Investigation of the effects of Chinese medicine on fibroblast viability: implications in wound healing," Phytotherapy Research, vol. 21, no. 10, pp. 938-947, 2007.

[85] T. W. Lau, Y. W. Chan, C. P. Lau et al., "Radix astragali and Radix rehmanniae, the principal components of two antidiabetic foot ulcer herbal formulae, elicit viability-promoting effects on primary fibroblasts cultured from diabetic foot ulcer tissues," Phytotherapy Research, vol. 23, no. 6, pp. 809-815, 2009.

[86] L. Tang, L. Liu, and D. S. Xu, "Preparation and purification of oligosaccharide from Radix rehmanniae," Chinese Traditional and Herbal Drugs, vol. 39, no. 8, pp. 1167-1171, 2008.
[87] R. X. Zhang, M. X. Li, and Z. P. Jia, "Rehmannia glutinosa: review of botany, chemistry and pharmacology," Journal of Ethnopharmacology, vol. 117, no. 2, pp. 199-214, 2008.

[88] O. Romero-Cerecero, A. Zamilpa-Álvarez, A. Ramos-Mora et al., "Effect on the wound healing process and in vitro cell proliferation by the medicinal mexican plant Ageratina pichinchensis," Planta Medica, vol. 77, no. 10, pp. 979-983, 2011.

[89] O. Romero-Cerecero, A. Zamilpa, M. González-Cortazar et al., "Pharmacological and chemical study to identify woundhealing active compounds in Ageratina pichinchensis," Planta Medica, vol. 79, no. 8, pp. 622-627, 2013.

[90] B. P. Muley, S. S. Khadabadi, and N. B. Banarase, "Phytochemical constituents and pharmacological activities of Calendula officinalis Linn (Asteraceae): a review," Tropical Journal of Pharmaceutical Research, vol. 8, no. 5, 2009.

[91] Committee on Herbal Medicinal Products (HMPC), “Assessment report on Olea europaea L., folium," European Medicines Agency, vol. 44, pp. 1-6, 2011.

[92] G. C. Gurtner, S. Werner, Y. Barrandon, and M. T. Longaker, "Wound repair and regeneration," Nature, vol. 453, no. 7193, pp. 314-321, 2008.

[93] L. Martinez and B. PachecoII, "Morfometria de fibroblastos e fibrócitos durante o processo cicatricial na pele de coelhos da raça Nova Zelândia Branco tratados com calêndula," Ciência Rural, vol. 38, no. 6, pp. 1662-1666, 2008.

[94] V. Duran, M. Matic, M. Jovanovc et al., "Results of the clinical examination of an ointment with marigold (Calendula officinalis) extract in the treatment of venous leg ulcers," International Journal of Tissue Reactions, vol. 27, no. 3, pp. 101-106, 2005.

[95] M. J. Leach, "Calendula officinalis and wound healing: a systematic review," Wounds: A Compendium of Clinacal Research and Practise, vol. 20, no. 8, pp. 236-243, 2008. 


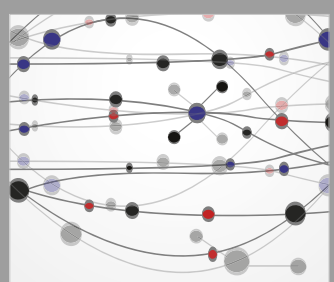

The Scientific World Journal
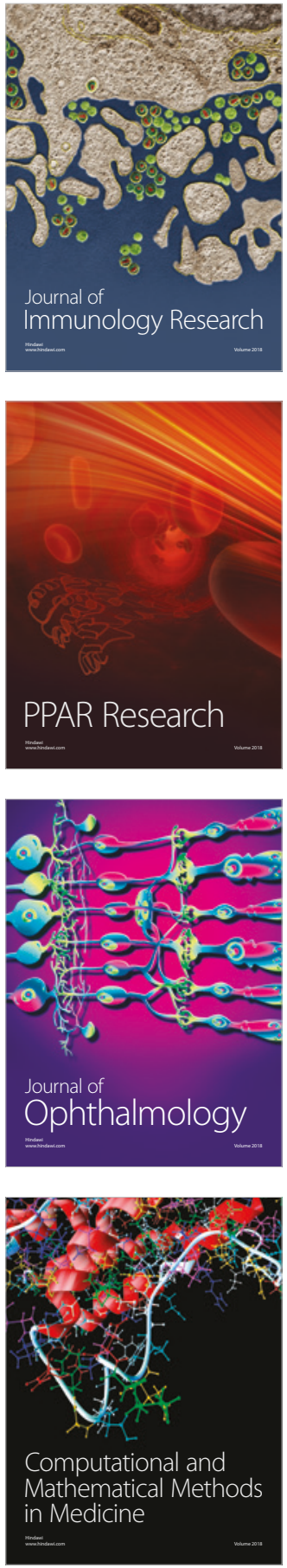

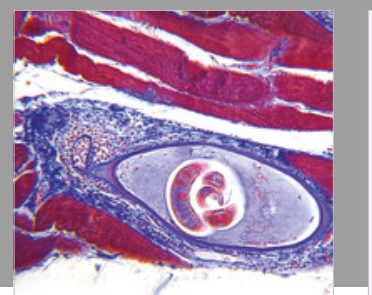

Gastroenterology Research and Practice

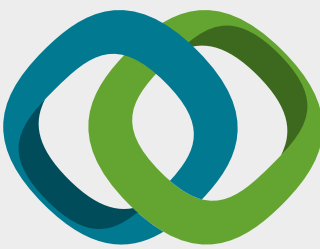

\section{Hindawi}

Submit your manuscripts at

www.hindawi.com
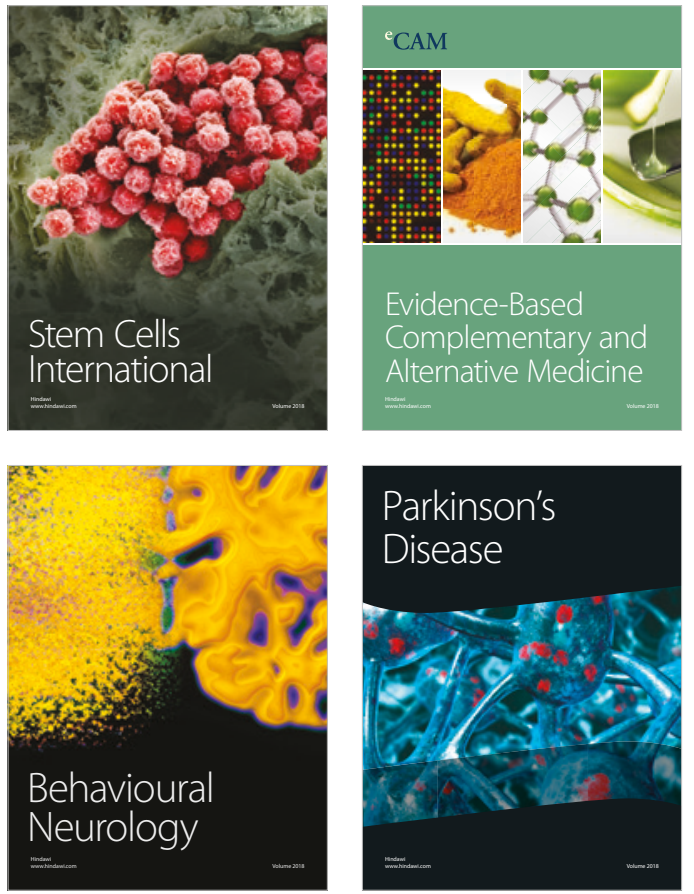

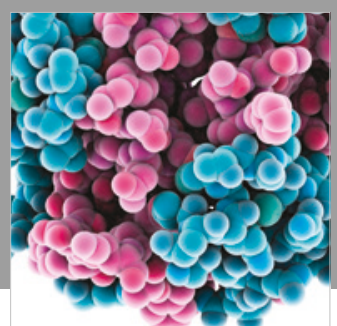

ournal of

Diabetes Research

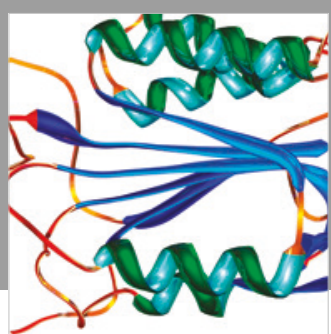

Disease Markers
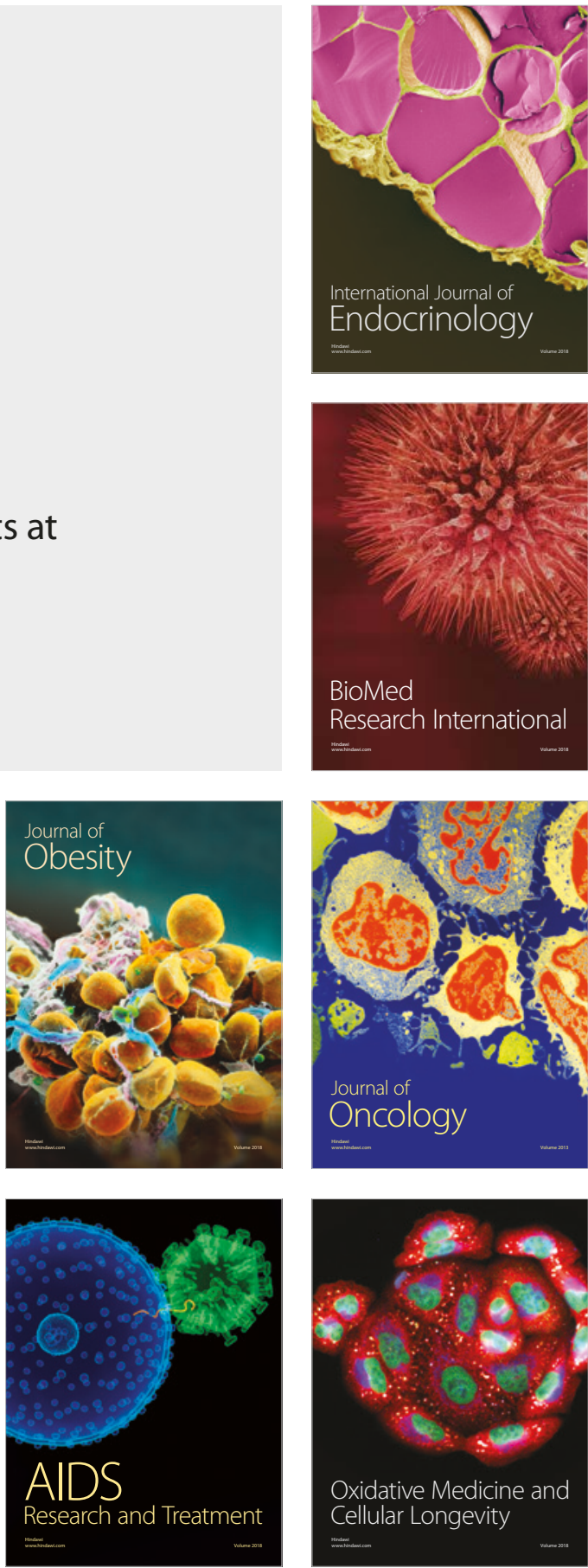